\title{
Antibiotics Use Patterns for Surgical Prophylaxis Site Infection in Different Surgical Wards of a Teaching Hospital in Ahvaz, Iran
}

\author{
Seyed Mohammad Alavi ${ }^{1}$; Fatemeh Roozbeh ${ }^{2}$; Farzaneh Behmanesh ${ }^{2}$; Leila Alavi ${ }^{3,}$ \\ ${ }^{1}$ Health Institute, Infectious and Tropical Disease Research Center, Ahvaz Jundishapur University of Medical Sciences, Ahvaz, IR Iran \\ ${ }^{2}$ Faculty of Medicine, Ahvaz Jundishapur University of Medical Sciences, Ahvaz, IR Iran \\ 3 Biochemical laboratory, Food and Drug Deputy, Ahvaz Jundishapur University of Medical Sciences, Ahvaz, IR Iran \\ ${ }^{*}$ Corresponding author: Leila Alavi, Food and Drug Deputy, Ahvaz Jundishapur University of Medical Sciences, Ahvaz, IR Iran. Tel: +98-6113387724, Fax: +98-6113335396, E-mail: leila. \\ alavi87@yahoo.com
}

Received: June 29, 2013; Revised: December 28, 2013; Accepted: January 14, 2014

\begin{abstract}
Background: Despite the effectiveness of prophylactic antimicrobials to prevent surgical site infection the use of antibiotic prophylaxis is often inappropriate.

Objectives:The current study aimed to determine the pattern of prophylactic antibiotic use ina teaching hospital affiliated to Jundishapur University of Medical Sciences, Ahvaz, Iran.

Patients and Methods: The current descriptive study included 8586 patients who received prophylactic antibiotics before surgery from April 2011 to March 2012, in Razi Hospital affiliated to Jundishapur University of Medical Sciences. Indications for antibiotic use, proper or inappropriate antibiotics, an antibiotic or combination of antibiotics, dosage and length of treatment for each patient based on the infectious disease textbook (Mandel's Principle and practice of infectious diseases) definitions were administrated.

Results: Of the total 8586 patients who took antibiotics for preventive purposes, 4815 (56\%) required antimicrobial prophylaxis, and 3771 (44\%) patients did not. Of the 4815 patients who received prophylaxis, 86.9\% received it appropriately,13.1\% received it inappropriately; $8.2 \%$ received inappropriate dosage, and 9.5\% received antibiotic longer than 24 hours.

Conclusions: The current study revealed that $44 \%$ of those who received prophylaxis did not need it. In the patients who received antibiotics, the most common mistakes were antibiotic selection followed by prolonged prophylaxis ( $>24$ hours) and excess dose.
\end{abstract}

Keywords:Prophylaxis; Infection; Nosocomial Infection

\section{Background}

In the recent years, in response to the medical improvements, the field of infection control has progressed by cooperation of the epidemiology with health sciences to decrease risk factors for health care-associated infections (HAls); therefore, interventions to prevent HAls should be implemented. The primary role of an infection-control program is to reduce the risk of nosocomial infection through protecting patients, employees, health care students, and visitors (1). Health care-associated infections are reported in 1.7 million cases annually in the United States with approximately 100,000 deaths (2).

The functions of a hospital epidemiology program vary from institution to institution, but can be generally divided into the following areas: surveillance, outbreak investigation, education, employee health, the monitoring and management of institutional antibiotic use and antibiotic resistance, the development of infectioncontrol policies and procedures, environmental hygiene, and new-product evaluation. Nearly $60 \%$ of the admitted patients receive antibiotics, and antibiotic usage varies widely across hospitals (3). The hospital epidemiology program should monitor the current antimicrobial status and susceptibility profiles on a regular basis to observe the trends in the development of antibiotic resistance. The results should be adjusted with the antimicrobial agents currently used in the hospital.

The best data are obtained if prophylactic antibiotics are distinguished from antibiotics used for treatment. Efforts should be made to optimize antimicrobial prophylaxis for surgical procedures, optimize selection of the first choice antibiotic and duration of empiric antimicrobial therapy, and improve antimicrobial prescribing practices. A variety of approaches can be undertaken including educational, administrative, e g formulary restrictions, and direct interventions by a team that manages antimicrobial use in the real time (4). Post operation wound infection is a major cause of morbidity, mortality, and health care costs. A considerable proportion of the millions of operations in the world each year are complicated by a surgical site infection (SSI) (5).

Mortality rates and hospital readmission rates are significantly increased. Post-surgery infections increase the length of stay and charges. When an SSI occurs, hospital readmissions and health care services at home increase (6).

Copyright (C) 2014,Ahvaz Jundishapur University of Medical Sciences; Published by Kowsar. This is an open-access article distributed under the terms of the Creative Commons Attribution-NonCommercial 4.0 International License (http://creativecommons.org/licenses/by-nc/4.0/) which permits copy and redistribute the material just in noncommercial usages, provided the original work is properly cited. 
The effectiveness of prophylactic antimicrobials to prevent SSIs was established in the 1960s. The use of antibiotics to prevent postoperative infections plays an important role in reducing the incidence of postoperative infections, duration of stay in hospital, cost of treatment, mortality, and helps the patient go back to the normal life more quickly (7). Locally published studies (in Farsi) indicated that antibacterial agents, ranked second after analgesic drugs in Iran and the consumption of these drugs in the country shows positive trend over the years. Although it was expected that the new antibiotics reduce the number of deaths and the incidence of infectious diseases, in practice they did not $(8,9)$. Indeed, increasing the incidence of infectious diseases caused by bacterial resistance against antibiotics, emerging and re-emerging of infectious diseases, and mortality of nosocomial infections are mostly due to the incorrect use of antibiotics that is a major health problem in the country $(8,10,11)$. However, despite the effectiveness of prophylactic antimicrobials to prevent SSIs, the use of antibiotic prophylaxis is often inappropriate.

Previous reports indicated that timing of administration, selection of the antibiotic, and duration of prophylaxis were inappropriate in the great majorities of cases $(5,7,12-16)$. While antibiotic prophylaxis is common in surgical procedures, inappropriate use of antibiotics occurs in $25 \%$ to $50 \%$ of the general elective surgeries (17). Unnecessary use of antibiotics and prolonged antibiotic prophylaxis (more than 48 hours) are significantly associated with increased risk of antimicrobial resistant microorganisms (7).

\section{Objectives}

To establish strategies for rational use of antibiotics to prevent SSI in hospitals, it is necessary to be aware of the current status of antibiotic use patterns. Searching the database of Ahvaz University of Medical Sciences (published dissertations and articles) shows that official reports or studies in this field in Khuzestan province do not exist; therefore, the current study aimed to determine the prophylactic antibiotic use patterns in a teaching hospital affiliated to Jundishapur University of Medical Sciences.

\section{Patients and Methods}

\subsection{Study Design}

Simple descriptive study.

\subsection{Study Time and Place}

From April 2011 to March 2012, this study performed in Razi Hospital affiliated to Ahvaz Jundishapur University of Medical Sciences.

\subsection{Definitions}

According to Mangram's guideline, post-surgical wounds are categorized as: Class 1: Clean Wound; An un- infected operative wound in which no inflammation is encountered and the respiratory, alimentary, genital, or uninfected urinary tract is not entered. In addition, clean wounds are primarily closed and, if necessary, drained with closed drainage. Operative incisional wounds that follow non penetrating (blunt) trauma should be included in this category if they meet the criteria. Class 2: Cleancontaminated Wound; it is an operative wound in which the respiratory, alimentary, genital or urinary tracts are entered under controlled conditions and without unusual contamination. Specifically, operations involving the biliary tract, appendix, vagina, and oropharynx are included in this category, provided that no evidence of infection or major break in technique is encountered. Class3: Contaminated Wound; open, fresh, accidental wounds. In addition, operations with major breaks in sterile technique, e.g. open cardiac massage, or gross spillage from the gastrointestinal tract, and incisions in which no acute purulent inflammation is encountered are included in this category. Class 4: Dirty-Infected Wound; that is old traumatic wounds with retained devitalized tissue and those that involve the existing clinical infection or perforated viscera. This definition suggests that the organisms causing postoperative infection were present in the operative field before the operation (18). In the current study, based on the extracted data from medical files, the patients possessing the inclusion criteria were reviewed as cases.

Procedure was defined as clean if no inflammation was encountered and the respiratory, alimentary, genital, or infected urinary tract was not entered. Procedure was defined as clean-contaminated if genital, alimentary respiratory or infected urinary tract was entered. Operations involving the appendix, biliary tract, vagina and oropharynx are also included in clean-contaminated procedures. The procedure was dirty-infected if clinical infection existed or devitalized tissue was observed in the old traumatic wounds. Conditions other than the mentioned ones were considered as contaminated cases. Prophylactic antibiotic was necessary for all clean-contaminated procedures and certain clean procedures, that is the ones in which intravascular/joint prosthetic device was inserted or there were risk factors, i.e. diabetes mellitus, malnutrition, obesity, irradiation history, use of immunosuppressant, or smoking. Dirty or contaminated procedures under treatment of antibiotics were not considered prophylaxis. Antibiotic selection was appropriate if selection criteria were available. This criterion was as follows:

Cefazolin is the selected antibiotic for cardiac, orthopedic, vascular, and neurosurgery operations; where Methicillin Resistant Staphylococcus aureus (MRSA) is suspected to cefazolin, the study shifted to vancomycin and in the case of anaerobic pathogens exist, metronidazole was added. Dose and timing of antibiotic is defined as proper if infusion of one to two grams cefazolin, one gram vancomycin or $500 \mathrm{mg}$ metronidazole started 60 minutes before incision and repeated three to five hours cefazo- 
Alavi SM et al.

lin, six to eight hours metronidazole, or 12 hours vancomycin after the first dose. Duration of prophylaxis was considered sufficient if lasted less than 24 hours, except 48 hours for cardiac surgery.

\subsection{Study Population}

The 8586 patients who received prophylactic antibiotics before surgery were enrolled. Patients were studied according to the type of surgery, the department they were admitted in, and the regimen of antibiotic prophylaxis. All variables were analyzed including demographic characteristics, underlying diseases, type of illness, kind of surgery, received antibiotics, the mode and dose of antibiotic, and the length of treatment. Indications for antibiotic use, proper or inappropriate antibiotics, an antibiotic or combination of antibiotics, dosage, and the length of treatment for each patient were administrated, based on the abovementioned definitions in the textbook of infectious diseases (7).

\subsection{Statistics}

The data were analyzed by SPSS-16 software using descriptive statistics.

\section{Results}

Of the total 13763 patients $47.5 \%$ and $52.5 \%$ were male and female respectively, 8945 procedures were clean, 3440 were clean-contaminated, 963 were contaminated and 412 were dirty. The 8586 patients underwent preoperative antibiotic prophylaxis. Of the total 8586 patients who took antibiotics for preventive purposes, 4815 (56\%) required antimicrobial prophylaxis, and 3771 (44\%) patients did not. Unnecessary use of prophylactic antibiotics was observed in 3771 (44\%) patients. Of the total 4815 medical procedures requiring antimicrobial prophylaxis, all received it. Of the 4815 patients who received perioperative prophylaxis, $4182(86.9 \%)$ cases received it appropriately, and $633(13.1 \%)$ received it inappropriately, 397 (8.2\%) cases received inappropriate dosage, and 457 (9.5\%) cases received antibiotic longer than 24 hours.

Certain patients received both incorrect drug dosage and duration. Characteristics of procedures are shown in Table 1. The used antibiotics are shown in Figure 1. Cefazolin, vancomycin, gentamicin, and metronidazole were the most commonly used drugs for prophylactic purposes. The frequency of patients who received cefazolin in the departments of obstetrics, general surgery, and orthopedics was 4261,2457 , and 2322 , respectively. The frequency of patients who received vancomycin in the departments of obstetrics, general surgery, and orthopedics was 2025,820 , and 705 , respectively. The number of patients who received gentamicin in the departments of obstetrics, general surgery and orthopedics was 1152 , 2300 , and 1025 respectively, and the number patients who received metronidazole in the departments of obstetrics and general surgery was 512 and 1750, respectively.
The frequency of patients who received these antibiotics is shown in Figure 1. Antimicrobial selection, cefazolin, vancomycin, and metronidazole, was appropriate in most of the cases except for gentamicin. Unnecessary use of gentamicin was observed in all surgery departments. Comparison of the hospital departments for prophylaxis and unnecessary prophylaxis are shown in Tables 2 and 3. Comparison of the hospital departments for incorrect antibiotic regimen, inappropriate dosage of antibiotic, and the length of treatment are shown in Table 4 . The most unnecessary antibiotic use was observed in general surgery ward and the most inappropriate antibiotic regimen, wrong dosage, and length of antibiotics were observed in obstetrics ward.

\begin{tabular}{|c|c|c|c|c|}
\hline \multirow[t]{2}{*}{ Characteristic } & \multicolumn{3}{|c|}{ Surgery Department } & \multirow[t]{2}{*}{ Total } \\
\hline & Obstetrics & General & Orthopedic & \\
\hline Clean & 5639 & 1725 & 1581 & 8945 \\
\hline $\begin{array}{l}\text { Clean-Contami- } \\
\text { nated }\end{array}$ & 2169 & 663 & 608 & 3440 \\
\hline Contaminated & 607 & 185 & 170 & 962 \\
\hline Dirty & 261 & 81 & 74 & 416 \\
\hline
\end{tabular}

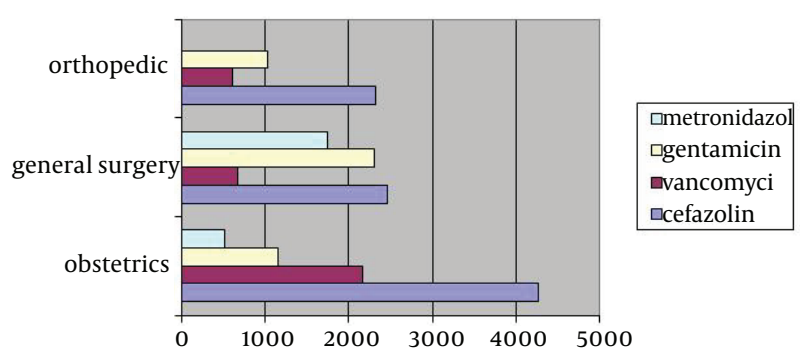

Figure 1. Antibiotics Used in the Surgery Departments

Table 2. Patients Admitted in the Surgery Departments ${ }^{a}$

Department Obstetrics General Orthopedic Total

\begin{tabular}{lllll}
\hline Prophylaxis & $3906(45)$ & $2357(88.8)$ & $2323(95.5)$ & $8586(62.4)$
\end{tabular}

Total admit- $8676(100) \quad 2654(100) \quad 2433(100) \quad 13763(100)$ ted patients

${ }^{\mathrm{a}}$ Data are presented as No.(\%).

Table 3. Unnecessary Use of Prophylactic Antibiotics in Patients Admitted in Surgery Departments a

\begin{tabular}{lcccc}
\hline Department & Obstetrics & General & Orthopedic & Total \\
\hline $\begin{array}{l}\text { Unneces- } \\
\text { sary use of } \\
\text { antibiotics }\end{array}$ & $1757(44.9)$ & $1084(44.4)$ & $930(40.0)$ & $3771(44)$ \\
$\begin{array}{l}\text { Total Pro- } \\
\text { phylaxis }\end{array}$ & $3906(100)$ & $2357(100)$ & $2323(100)$ & $8586(100)$ \\
\hline
\end{tabular}

${ }^{\mathrm{a}}$ Data are presented as No. (\%). 
Alavi SM et al.

\begin{tabular}{lcccc}
\hline Table 4. Inappropriate Antibiotics Selection, Dosage, and Length of Treatment in Patients Admitted in Surgery Departments a \\
\hline Department & Obstetrics & General & Orthopedic & Total \\
\hline Inappropriate antibiotic regimen & $363(16.9)$ & $204(16.1)$ & $66(4.8)$ & $633(13.1)$ \\
Inappropriate dosage & $195(9.1)$ & $120(9.5)$ & $82(5.9)$ & $397(8.2)$ \\
Insufficient treatment duration & $292(13.6)$ & $28(2.2)$ & $137(9.8)$ & $457(9.5)$ \\
Total antibiotic indicated cases & $2149(100)$ & $1273(100)$ & $1393(100)$ & $4815(100)$ \\
\hline
\end{tabular}

${ }^{\mathrm{a}}$ Data are presented as No. (\%).

\section{Discussion}

Of the six infection prevention measures, three core measures contain recommendations regarding the selection of prophylactic antibiotics, timing of administration, and duration of therapy (12). In the current study, for most of the patients undergoing clean-contaminated surgeries, gastrointestinal, orthopedic, and gynecologic, infection control committee recommended an antibiotic regimen including the first generation cephalosporin such as cefazolin or vancomycin, when MRSA infection is suspected; with or without metronidazole, if anaerobic pathogens were likely, as the choice antibiotic regimen to prevent post operation infections. Results of the current study revealed that the current patterns of using antibiotics to prevent surgical infections in Razi Hospital were not favorable. In total, approximately $45 \%$ of antibiotics were prescribed without indication and were unnecessary.

Approximately 13\% (8.2\% to $13 \%$ ) of the indicated antibiotic prophylaxis was inappropriate at least in antibiotic regimen, dose, or duration of antibiotic administration. In spite of hospital infection control recommendations, the application of prophylactic antimicrobials to prevent SSIs and use of antibiotic prophylaxis were often inappropriate. Published studies indicate that the antibiotic regimen selection, timing of administration, dose of antibiotic and duration of prophylaxis in majority of the cases is inappropriate $(5,7,12-16)$. For example it is administered when not required, not administered when required, or not administered properly $(7,15)$. In contrast to the findings of other studies, however, the prescription of antibiotics in Iranian hospitals is irrational, it is not higher than those of the other regions of the world, but it is still unacceptable.

The infection control committee in Razi Hospital has not distributed a guideline for surgical prophylaxis based on the standard documents and original researches conducted by microbiology department of the university regarding the most common isolated organisms and susceptibility patterns. It seems advisable that surgeons consult with infectious diseases specialist before administration of antibiotics for prophylaxis for surgical procedures in special clinical situations (19)

The present study found that approximately $42 \%$ of the studied patients received appropriate antimicrobial prophylaxis. Prophylaxis was inappropriately performed in
$13 \%$ of 4,815 patients requiring prophylaxis. In the current study, 3771 (about 44\%) patients received preoperative antibiotic, but they did not need it. Thus, the most common wrong activities of surgical prophylaxis in Razi Hospital appear to be excessive use of antibiotics. This finding is consistent with reports from developing countries as well as Iran $(16,20,21)$, but in contrast to developed country where the most common error in the surgical prophylaxis practice is omission of antibiotics rather than their excessive use (15). The current study also found that all of the patients under study received antibiotics on time. As a routine in the hospital, initiation of prophylaxis is at the time of anesthesia induction. This finding is similar to that of the studies performed in other areas $(15,16)$.

The choice of antimicrobial compounds was appropriate in approximately $87 \%$ of the cases. However, six different drugs or drug combinations were used; cefazolin, cefazolin plus gentamicin, vancomycin, vancomycin plus gentamycin, cefazolin plus metronidazole, and vancomycin plus metronidazole. Excessive use of vancomycin and unnecessary use of gentamicin in the study is problematic. This problem is similar to that of a study previously performed $(16,22)$. Appropriate selection of antibiotics reported by Vaisbrud et al. (15) was 95\%, but that of the current work was $86.9 \%$. Surgeons` unawareness to consult with infectious disease specialists and lack of certain guidelines of antibiotics use for prophylaxis in the hospital may be discussed for these differences. In the current study, duration of antibiotics administration was appropriate in $90.5 \%$.

The rate of longer duration of administration of antibiotics was 9.5\%, which ranged from $2.2 \%$ in general surgery department to $13.6 \%$ in gynecology department. This finding was inconsistent with that of Vaisbrud et al. with $91 \%$ (15). After unnecessary use of antibiotics and selection of antibiotics, the most commonly detected error was too long postoperative administration of antibiotics. Majority of Iranian surgeons insist on the need for prolonged postoperative use of antibiotic therapy to prevent postoperative infections. It is known well that prolonged use of post-operative antimicrobials not only does not provide additional benefit, but also affects emerging the bacterial resistance (7). The current study had limitations such as retrospective design, restriction to just one hospital, and only three surgery departments. Since the condi- 
tion of all hospitals affiliated to Jundishapur University is similar from the study purposes view point; therefore, restriction to a hospital could not result in significant bias. In conclusion, the current study revealed that about $42 \%$ of patients received appropriate prophylactic antibiotics. However, $44 \%$ of those who received prophylaxis did not need it. In patients receiving antibiotics, the most common mistakes were antibiotic selection followed by prolonged prophylaxis ( $>24$ hours), and excess dose of antibiotics. Feeding this information back to surgeons and establishing pre-operative prophylaxis guidelines as a routine activity of infection control teams could improve nosocomial infection control program.

\section{Acknowledgements}

This article is derived from medical files of Razi Hospital. The authors greatly appreciate the chief and medical archivists of Razi Hospital. The authors also thank the staff of Jundishapur Infectious Diseases Research Center for their kind cooperation.

\section{Authors' Contributions}

Study concept and design: SM Alavi. Analysis and interpretation of data: L Alavi,. Drafting of the manuscript: SM Alavi, Roozbeh, Behmanesh. Critical revision of the manuscript for important intellectual content: Roozbeh, Behmanesh, and L Alavi. Statistical analysis: L Alavi.

\section{Funding/Support}

This study is supported by Jundishapur Infectious and Tropical Diseases Research Center.

\section{References}

1. Savage JW, Anderson PA. An update on modifiable factors to reduce the risk of surgical site infections. Spine J. 2013;13(9):1017-29.

2. Klevens RM, Edwards JR, Richards CL, Jr, Horan TC, Gaynes RP, Pollock DA, et al. Estimating health care-associated infections and deaths in U.S. hospitals, 2002. Public Health Rep. 2007;122(2):160-

3. MacDougall C, Polk RE. Variability in rates of use of antibacterials among 130 US hospitals and risk-adjustment models for interhospital comparison. Infect Control Hosp Epidemiol. 2008;29(3):203-11.

4. MacDougall C, Polk RE. Antimicrobial stewardship programs in health care systems. Clin Microbiol Rev. 2005;18(4):638-56.

5. DeFrances CJ, Cullen KA, Kozak LJ. National Hospital Discharge Survey: 2005 annual summary with detailed diagnosis and pro- cedure data. Vital Health Stat 13. 2007(165):1-209.

6. Astagneau P, Rioux C, Golliot F, Brucker G. Morbidity and mortality associated with surgical site infections: results from the 19971999 INCISO surveillance. J Hosp Infect. 2001;48(4):267-74.

7. Talbot TR. Surgical Site Infections and Antimicrobial Prophylaxis. In: Mandell GL, Bennett JE, Dolin R editors. Principle and practice of infectious diseases. 7th ed. Philadelphia: Churchill Livingstone; 2010. pp. 3890-905.

8. Engemann JJ, Carmeli Y, Cosgrove SE, Fowler VG, Bronstein MZ, Trivette SL, et al. Adverse clinical and economic outcomes attributable to methicillin resistance among patients with Staphylococcus aureus surgical site infection. Clin Infect Dis. 2003;36(5):592-8.

9. Bratzler DW, Hunt DR. The surgical infection prevention and surgical care improvement projects: national initiatives to improve outcomes for patients having surgery. Clin Infect Dis. 2006;43(3):322-30.

10. Mera RM, Miller LA, Daniels JJ, Weil JG, White AR. Increasing prevalence of multidrug-resistant Streptococcus pneumoniae in the United States over a 10-year period: Alexander Project. Diagn Microbiol Infect Dis. 2005;51(3):195-200.

11. Neuhauser MM, Weinstein RA, Rydman R, Danziger LH, Karam G, Quinn JP. Antibiotic resistance among gram-negative bacilli in US intensive care units: implications for fluoroquinolone use. JAMA. 2003;289(7):885-8.

12. National Nosocomial Infections Surveillance S. National Nosocomial Infections Surveillance (NNIS) System Report, data summary from January 1992 through June 2004, issued October 2004. Am J Infect Control. 2004;32(8):470-85.

13. Fonseca LG, de Oliveira Conterno L. Audit of antibiotic use in a Brazilian University Hospital. Braz J Infect Dis. 2004;8(4):272-80.

14. Salkind AR, Rao KC. Antiobiotic prophylaxis to prevent surgical site infections. Am Fam Physician. 2011;83(5):585-90.

15. Vaisbrud V, Raveh D, Schlesinger Y, Yinnon AM. Surveillance of antimicrobial prophylaxis for surgical procedures. Infect Control Hosp Epidemiol. 1999;20(9):610-3.

16. Yalcin AN, Serin S, Gurses E, Zencir M. Surgical antibiotic prophylaxis in a Turkish university hospital.J Chemother. 2002;14(4):373-7.

17. Bedouch P, Labarere J, Chirpaz E, Allenet B, Lepape A, Fourny M, et al. Compliance with guidelines on antibiotic prophylaxis in total hip replacement surgery: results of a retrospective study of 416 patients in a teaching hospital. Infect Control Hosp Epidemiol. 2004;25(4):302-7.

18. Mangram AJ, Horan TC, Pearson ML, Silver LC, Jarvis WR. Guideline for prevention of surgical site infection, 1999. Hospital Infection Control Practices Advisory Committee. Infect Control Hosp Epidemiol. 1999;20(4):250-78.

19. Schlesinger Y, Paltiel O, Yinnon AM. Analysis and impact of infectious disease consultations in a general hospital. J Hosp Infect. 1998;40(1):39-46.

20. Finkelstein R, Reinhertz G, Embom A. Surveillance of the use of antibiotic prophylaxis in surgery. Isr J Med Sci. 1996;32(11):1093-7.

21. Lizan-Garcia M, Garcia-Caballero J, Asensio-Vegas A. Risk factors for surgical-wound infection in general surgery: a prospective study. Infect Control Hosp Epidemiol. 1997;18(5):310-5.

22. Kurz X, Mertens R, Ronveaux O. Antimicrobial prophylaxis in surgery in Belgian hospitals: room for improvement. Eur J Surg. 1996;162(1):15-21. 